\title{
Fatty acids and Alzheimer's Disease: Evidence on Cognition and Cortical $\beta$-amyloid from Secondary Analyses of the Multidomain Alzheimer Preventive Trial
}

\author{
C. Hooper ${ }^{1}$, B. Vellas $^{1,2}$ for the MAPT/DSA study group \\ 1. Gérontopôle, Department of Geriatrics, CHU Toulouse, Purpan University Hospital, Toulouse, France; 2. MR1027, Université de Toulouse, UPS, INSERM, Toulouse, \\ France
}

Corresponding Author: Claudie Hooper, Gérontopôle, Department of Geriatrics, CHU Toulouse, Purpan University Hospital, Toulouse, France. claudie28@yahoo.com, Tel : +33 (5) 617764 25, Fax : +33 (5) 61776475

J Prev Alz Dis 2018;5(3):168-170

Published online February 20, 2018, http:/ /dx.doi.org/10.14283/jpad.2018.7

\section{Fatty acids and the brain}

Datty acids are long-chain hydrocarbons that 4 can be separated into four groups: saturated, L monounsaturated, polyunsaturated, and trans fats (1). The brain is highly enriched in fatty acids particularly polyunsaturated fatty acids (PUFAs) with docosahexaenoic acid (an omega 3: n-3 PUFA) and arachidonic acid (an omega 6: n-6 PUFA) being the most abundant $(2,3)$. Fats control the structure and function of cell membranes and therefore impact upon signal transduction and neurotransmission and PUFAs play a role in inflammatory processes (4). Saturated and monounsaturated fatty acids can be synthesized de novo within the brain, but PUFAs are mainly supplied by the blood (5).

\section{The Multidomain Alzheimer Preventive Trial}

The Multidomain Alzheimer Preventive Trial (MAPT) was a large phase III, 3 year, multicentre, randomized, placebo-controlled trial (registration: NCT00672685) (6). The trial had four arms comprising a placebo group and three treatment groups: n-3 PUFA supplementation (docosahexaenoic acid + eicosapentaenoic acid), multidomain intervention (involving nutritional and exercise counselling and cognitive training) and $n-3$ PUFA supplementation plus multidomain intervention. The trial was designed to assess the efficacy of the interventions in slowing cognitive decline in older adults at risk of dementia $(n=1680)(6)$. The main analysis of MAPT showed no significant effects of any of the interventions on cognitive function compared to placebo after adjustment for multiple testing (7).

\section{MAPT [18F] Florbetapir Positron Emission Tomography ancillary study}

Subjects participated in the MAPT [18F] florbetapir positron emission tomography (PET) ancillary study on a voluntary basis $[\mathrm{n}=271: \mathrm{n}=70(25.8 \%)$ placebo, $\mathrm{n}=60(22.1 \%) \mathrm{n}-3$ PUFA supplementation, $\mathrm{n}=68$ $(25.1 \%)$ multidomain intervention, $\mathrm{n}=73(26.9 \%)$ multidomain intervention and n-3 PUFA]. PET scans were performed using [18F] florbetapir to provide a measure of cerebral $\beta$-amyloid $(A \beta)$ load $(6,8)$. Regional standard uptake value ratios (SUVRs) were generated from semi-automated quantitative analysis with the whole cerebellum as the reference region. Cortical-tocerebellar SUVRs (cortical-SUVRs) were obtained using the mean signal of the following predefined cortical regions: frontal, temporal, parietal, precuneus, anterior cingulate, and posterior cingulate as previously described (9).

\section{Fats and cognition: evidence from secondary exploratory analyses of MAPT data}

Although, in the main analysis of MAPT it was shown that n-3 PUFA supplementation was not effective against cognitive decline (7), subsequent within group analysis has shown that subjects in the placebo group of MAPT with a low omega 3 index (defined as $\leq 4.83 \%$ which represents the lowest quartile of omega 3 index distribution: $n=59$ at 3 years) declined significantly on a cognitive composite $Z$ score over 3 years (mean change -0.236 , SE $0.072, p<0.05$ ), whereas those on placebo with a higher omega-3 index (upper three quartiles) remained stable (mean change -0.011, SE, 0.037, p > 0.05) (10). The mean cognitive decline over three years in the subjects in the lowest quartile of omega 3 index distribution was comparable to the cognitive decline observed in MAPT subjects with a Clinical Dementia Rating (CDR) score of 0.5 (mean change $-0.184, \mathrm{SE}, 0.052, \mathrm{p}<0.05$ ) or carriers of the Apolipoprotein $\mathrm{E}$ (ApoE) $\varepsilon 4$ allele (mean change -0.208 , SE, 0.070, $\mathrm{p}<0.05$ (10).

Further exploratory analysis investigating the effects of n-3 PUFA supplementation in the MAPT subgroup with low omega-3 index $(\mathrm{n}=183$ including participants in the placebo and n-3 PUFA supplemented groups only) has shown that there was less decline on the Controlled 
Oral Word Association Test (COWAT: number of words/ 2 minutes) in the n-3 PUFA supplementation group compared to placebo $(\mathrm{p}=0.009$; between group mean difference over 3 years, 2.3; $95 \%$ CI, 0.6,4.0) (11). This suggests that n-3 PUFA supplementation might be beneficial over placebo for the maintenance of executive function (11). Non-memory changes such as changes in executive function are recognised as early cognitive changes in Alzheimer's disease (AD) (12). Collectively these findings from MAPT suggest that n-3 PUFA supplementation might prove therapeutically useful for the combat of cognitive changes in subjects with suboptimal n-3 PUFA levels in the early stages of AD.

\section{Fats and cortical $A \beta$ : evidence from secondary exploratory analyses of MAPT data}

At a preclinical level we have shown that erythrocyte membrane PUFAs were not associated with cortical $\mathrm{A} \beta$ using fully adjusted multiple regression models (13). This exploratory analysis was performed in MAPT subjects in the placebo group with data on cortical $A \beta$ and erythrocyte membrane PUFA levels $(n=61)$. These findings suggest that $n-3$ PUFAs might confer their beneficial effects on cognition via $A \beta$-independent pathways, although it would be interesting to examine whether n-3 PUFAs might be associated with $A \beta$ in subjects with sub-optimal n-3 PUFA levels. However, we did not have sufficient subjects to test this in MAPT.

In our exploratory analyses concerning cortical $\mathrm{A} \beta$, the associations closest to significance were those between erythrocyte membrane arachidonic acid and $\mathrm{A} \beta$ (B coefficient, 0.03; $95 \% \mathrm{CI}, 0.00,0.06 ; \mathrm{p}=0.04$ ) and erythrocyte membrane linoleic acid (the precursor to arachidonic acid) and $\mathrm{A} \beta$ (B coefficient, $-0.02 ; 95 \% \mathrm{CI}$, $-0.04,0.00 ; p=0.09$ ) (13). These findings suggest that $n-6$ PUFAs might be important in terms of the regulation of amyloidogenesis. Interestingly, arachidonic acid was positively associated with $A \beta$ whereas linoleic acid was inversely associated with $\mathrm{A} \beta$. We hypothesised that low linoleic acid might reflect its increased conversion to arachidonic acid, which in turn might lead to increased pro-inflammatory eicosanoid formation in turn fuelling amyloidogenesis. However, this hypothesis warrants validation. Furthermore, we found that the association between arachidonic acid and cortical $A \beta$ appeared to be specific to ApoE $\varepsilon 4$ non-carriers (B coefficient, 0.03; $95 \%$ CI, 0.00, 0.06; $\mathrm{p}=0.03$ ) compared to ApoE $\varepsilon 4$ carriers (B coefficient, $0.02 ; 95 \% \mathrm{CI},-0.04,0.08 ; \mathrm{p}=0.57)(13)$.

In another exploratory study of MAPT data we have shown that erythrocyte membrane saturated and monounsaturated fatty acids were also not associated with cortical A $\beta$ using fully adjusted multiple linear regression models (14). This analysis was also restricted to MAPT subjects in the placebo group with data on cortical $A \beta$ load and erythrocyte membrane fatty acid levels ( $n=61$ : the same population as described above).
In this study the association closest to significance was that between erythrocyte membrane stearic acid (saturated fatty acid) and cortical $\mathrm{A} \beta$ (B coefficient, 0.03; $95 \% \mathrm{CI}, 0.00,0.05 ; \mathrm{p}=0.05)$. This positive association, although statistically non-significant, appeared to be stronger amongst ApoE $\varepsilon 4$ carriers (B coefficient, 0.04; $95 \% \mathrm{CI},-0.01,0.09 ; \mathrm{p}=0.08)$ compared to ApoE $\varepsilon 4$ noncarriers (B coefficient, 0.02; $95 \% \mathrm{CI},-0.01,0.05$; $\mathrm{p}=0.18$ ), which is in contrast to our findings with arachidonic acid. However, these preliminary findings require further confirmation. Stearic acid has been shown not to modulate $\alpha$-secretase-dependent cleavage of amyloid precursor protein in cultured neuronal-like cells (15), implying that stearic acid might indirectly facilitate $A \beta$ accumulation over time. Furthermore, at a clinical level, stearic acid has previously been associated with cognitive decline in the elderly (16).

\section{Conclusion}

Here we suggest that n-3 PUFA supplementation might be beneficial for the prevention of cognitive decline in the elderly with sub-optimal n-3 PUFA status in the early stages of AD. This might explain the discrepant results seen with clinical trials implementing n-3 PUFAs in the past, which have not focussed on participants with low n-3 PUFA levels at baseline (4). It might be that supplementation restores homeostatic levels thereby preventing cognitive decline and our preliminary findings suggest that this occurs via $A \beta$-independent mechanisms. In terms of amyloidogenesis, high erythrocyte membrane arachidonic acid and stearic acid and low linoleic acid appeared to be weakly associated with increased cortical $A \beta$, although it should be noted that statistical significance was not reached in our exploratory studies. Thus, dietary fats might play an important role in the pathophysiology of $A D$ via differing mechanisms. Further studies are warranted to elucidate the roles of fats in $A \beta$ plaque formation, tau phosphorylation and cognitive decline particularly as a function of ApoE $\varepsilon 4$ status.

Acknowledgments: Funding: “The MAPT study was supported by grants from the Gérontopôle of Toulouse, the French Ministry of Health (PHRC 2008, 2009), Pierre Fabre Research Institute (manufacturer of the omega-3 supplement), Exhonit Therapeutics SA, Avid Radiopharmaceuticals Inc and in part by a grant from the French National Agency for Research called "Investissements d'Avenir" $\mathrm{n}^{\circ}$ ANR-11-LABX-0018-01. The promotion of this study was supported by the University Hospital Center of Toulouse. The data sharing activity was supported by the Association Monegasque pour la Recherche sur la maladie d'Alzheimer (AMPA) and the UMR 1027 Unit INSERM-University of Toulouse III". Sponsor's role: None.

MAPT/DSA Group refers to MAPT Study Group: Principal investigator: Bruno Vellas (Toulouse); Coordination: Sophie Guyonnet ; Project leader: Isabelle Carrié ; CRA: Lauréane Brigitte ; Investigators: Catherine Faisant, Françoise Lala, Julien Delrieu, Hélène Villars ; Psychologists: Emeline Combrouze, Carole Badufle, Audrey Zueras ; Methodology, statistical analysis and data management: Sandrine Andrieu, Christelle Cantet, Christophe Morin; Multidomain group: Gabor Abellan Van Kan, Charlotte Dupuy, Yves Rolland (physical and nutritional components), Céline Caillaud, Pierre-Jean Ousset (cognitive component), Françoise Lala (preventive consultation), Bertrand Fougère (Toulouse). The cognitive component was designed in collaboration with Sherry Willis from the 
University of Seattle, and Sylvie Belleville, Brigitte Gilbert and Francine Fontaine from the University of Montreal. Co-Investigators in associated centres: JeanFrançois Dartigues, Isabelle Marcet, Fleur Delva, Alexandra Foubert, Sandrine Cerda (Bordeaux); Marie-Noëlle-Cuffi, Corinne Costes (Castres); Olivier Rouaud, Patrick Manckoundia, Valérie Quipourt, Sophie Marilier, Evelyne Franon (Dijon), Lawrence Bories, Marie-Laure Pader, Marie-France Basset, Bruno Lapoujade, Valérie Faure, Michael Li Yung Tong, Christine Malick-Loiseau, Evelyne CazabanCampistron (Foix); Françoise Desclaux, Colette Blatge (Lavaur); Thierry Dantoine, Cécile Laubarie-Mouret, Isabelle Saulnier, Jean-Pierre Clément, Marie-Agnès Picat, Laurence Bernard-Bourzeix, Stéphanie Willebois, Iléana Désormais, Noëlle Cardinaud (Limoges); Marc Bonnefoy, Pierre Livet, Pascale Rebaudet, Claire Gédéon, Catherine Burdet, Flavien Terracol (Lyon), Alain Pesce, Stéphanie Roth, Sylvie Chaillou, Sandrine Louchart (Monaco); Kristelle Sudres, Nicolas Lebrun, Nadège Barro-Belaygues (Montauban); Jacques Touchon, Karim Bennys, Audrey Gabelle, Aurélia Romano, Lynda Touati, Cécilia Marelli, Cécile Pays (Montpellier); Philippe Robert, Franck Le Duff, Claire Gervais, Sébastien Gonfrier (Nice); Yves Gasnier and Serge Bordes, Danièle Begorre, Christian Carpuat, Khaled Khales, Jean-François Lefebvre, Samira Misbah El Idrissi, Pierre Skolil, Jean-Pierre Salles (Tarbes). MRI group: Carole Dufouil (Bordeaux), Stéphane Lehéricy, Marie Chupin, Jean-François Mangin, Ali Bouhayia (Paris); Michèle Allard (Bordeaux); Frédéric Ricolfi (Dijon); Dominique Dubois (Foix); Marie Paule Bonceour Martel (Limoges); François Cotton (Lyon); Alain Bonafé (Montpellier); Stéphane Chanalet (Nice); Françoise Hugon (Tarbes); Fabrice Bonneville, Christophe Cognard, François Chollet (Toulouse). PET scans group: Pierre Payoux, Thierry Voisin, Julien Delrieu, Sophie Peiffer, Anne Hitzel, (Toulouse); Michèle Allard (Bordeaux); Michel Zanca (Montpellier); Jacques Monteil (Limoges); Jacques Darcourt (Nice). Medico-economics group: Laurent Molinier, Hélène Derumeaux, Nadège Costa (Toulouse). Biological sample collection: Christian Vincent, Bertrand Perret, Claire Vinel (Toulouse). Safety management : Pascale Olivier-Abbal. DSA Group: Sandrine Andrieu, Christelle Cantet, Nicola Coley.

Conflict of Interest: The authors declare no conflict of interest. There were no financial relationships with any organizations that might have an interest in the submitted work or no other relationships or activities that could appear to have influenced the submitted work.

Ethical Standards: The MAPT protocol was approved by the French Ethics Committee located in Toulouse (CPP SOOM II) and was authorised by the French Health Authority (Ministry of Health). Written consent was obtained from all participants.

\section{References}

1. White B. Dietary fatty acids. Am Fam Physician. 2009. 80(4):345-50.

2. Thomas MH, Pelleieux S, Vitale N, Olivier JL. Dietary arachidonic acid as a risk factor for age-associated neurodegenerative diseases: Potential mechanisms. Biochimie. 2016. 26.

3. Carlson SE, Colombo J. Docosahexaenoic Acid and Arachidonic Acid Nutrition in Early Development. Adv Pediatr. 2016. 63(1):453-71.

4. Hooper C., De Souto Barreto P., Pahor M., Weiner M., Vellas B. The relationship of omega 3 polyunsaturated fatty acids in red blood cell membranes with cognitive function and brain structure: A review focussed on Alzheimer's disease. Journal of prevention of Alzheimer's disease. J Prev Alzheimers Dis. 2017. 19.

5. Bazinet RP, Layé S. Polyunsaturated fatty acids and their metabolites in brain function and disease. Nat Rev Neurosci. 2014. 15(12):771-85.

6. Vellas B, Carrie I, Gillette-Guyonnet S, Touchon J, Dantoine T, Dartigues JF, et al. MAPT STUDY: A MULTIDOMAIN APPROACH FOR PREVENTING ALZHEIMER'S DISEASE: DESIGN AND BASELINE DATA. J Prev Alzheimers Dis. 2014. 1(1):13-22.

7. Andrieu S, Guyonnet S, Coley N, Cantet C, Bonnefoy M, Bordes S, et al. Effect of long-term omega 3 polyunsaturated fatty acid supplementation with or without multidomain intervention on cognitive function in elderly adults with memory complaints (MAPT): a randomised, placebo-controlled trial. Lancet Neurol. 2017. 16(5):377-389.

8. Del Campo N, Payoux P, Djilali A, Delrieu J, Hoogendijk EO, Rolland Y, et al. Relationship of regional brain $\beta$-amyloid to gait speed. Neurology. 2016. 86(1):36-43

9. Joshi AD, Pontecorvo MJ, Clark CM, Carpenter AP, Jennings DL, Sadowsky $\mathrm{CH}$, et al. Performance characteristics of amyloid PET with florbetapir F 18 in patients with alzheimer's disease and cognitively normal subjects. J Nucl Med Off Publ Soc Nucl Med. 2012. 53(3):378-84.

10. J.K Chhetri, P. de Souto Barreto, C. Cantet, M. Cesari, N. Coley, S. Andrieu, B. Vellas. Trajectory of the MAPT-PACC Preclinical Alzheimer Cognitive Composite in the placebo group of a randomized control trial: Results from the MAPT study: Lessons for further trials. J Prev Alzheimers Dis. 2017. (in press).

11. Hooper C., De Souto Barreto P., Coley N., Cantet C., Cesari M., Andrieu S. Vellas B. Cognitive changes with omega-3 polyunsaturated fatty acids in nondemented older adults with low omega-3 index. J Nutr Health Aging. 2017. 21, 988-993.

12. Kivipelto M, Mangialasche F, Ngandu T. Can lifestyle changes prevent cognitive impairment? Lancet Neurol. 2017. 16(5):338-9.

13. Hooper C, De Souto Barreto P, Payoux P, Salabert AS, Guyonnet S, Andrieu $S$, et al. Cross-sectional associations of cortical $\beta$-amyloid with erythrocyte membrane long-chain polyunsaturated fatty acids in older adults with subjective memory complaints. J Neurochem. 2017. 142, 589-596.

14. Hooper C., De Souto Barreto P., Payoux P., Salabert A. S., Guyonnet S., Andrieu S., Vellas B. Association of cortical B-amyloid with erythrocyte membrane monounsaturated and saturated fatty acids in older adults at risk of dementia. J Nutr Health Aging. 2017. 21(10):1170-1175.

15. Yang X, Sheng W, Sun GY, Lee JC-M. Effects of fatty acid unsaturation numbers on membrane fluidity and $\alpha$-secretase-dependent amyloid precursor protein processing. Neurochem Int. 2011. 58(3):321-9.

16. Heude B, Ducimetière P, Berr C, EVA Study. Cognitive decline and fatty acid composition of erythrocyte membranes--The EVA Study. Am J Clin Nutr. 2003. 77(4):803-8. 